\title{
Topographie und funktionelle Anatomie des Schultergelenks
}

\author{
Ulrich Rumschik, Bernd Wittner
}

\section{Zusammenfassung}

Die Absicht dieses Beitrags ist es, die Anatomie des Schultergelenks nach funktionellen Gesichtspunkten $\mathrm{zu}$ behandeln und dabei die anatomischen Strukturen herauszuarbeiten, welchen bei bestimmten Erkrankungen oder Verletzungen dieser Körperregion besondere Bedeutung zukommt. Diese Zielsetzung bringt es mit sich, dass, im Gegensatz zu den deskriptiven Abhandlungen in anatomischen Lehrbüchern, die Beschreibung der Schulteranatomie keineswegs vollständig erfolgt, sondern mit einer gewollten Gewichtung nach klinischer Relevanz.

\section{Vier Gelenke}

Von allen Gelenken des menschlichen Körpers hat das Schultergelenk den größten Bewegungsumfang. Diese Bewegungsfreiheit ist aber nicht ausschließlich der Konstruktion des Schultergelenks selbst, des glenohumeralen Gelenks, zuzuschreiben, sondern resultiert aus der bemerkenswerten Tatsache, dass sich die Gelenkpfanne durch Rotations- und Translationsbewegungen des Schulterblatts variabel einstellen lässt. Von der maximalen Abduktionsfähigkeit des Armes entfallen 120 Grad auf das Schultergelenk, die restlichen 60 Grad trägt die Rotation der Scapula bei. Beide Bewegungskomponenten verlaufen größten Teils parallel, so dass schon ab 30 Grad Abduktion eine Mitrotation der Skapula erfolgt.

OP-JOURNAL 2002; 18: 184-189

(c) Georg Thieme Verlag Stuttgart · New York
Die Beweglichkeit der Schulter wird durch das Zusammenspiel von 4 Gelenken ermöglicht.

Das Schulterblatt ist durch das Schlüsselbein als Strebepfeiler mit dem Rumpf verbunden. Diese Verbindung ist durch Ausbildung des Sternoklavikulargelenks am medialen Ende der Klavikula und durch das Akromioklavikulargelenk lateral sehr beweglich.

Das Schulterblatt selbst ist gut gegen die darunterliegende Thoraxwand verschieblich, was zur Bezeichnung thorakoskapulares Gelenk geführt hat, obwohl kein Gelenk im herkömmlichen Sinne vorliegt.

Insgesamt sind es also vier Gelenke (Abb.1), Articulatio sternoclavicularis, Art. acromioclavicularis, Art. thoracoscapularis und Art. glenohumeralis, welche die einzigartige Beweglichkeit der Schulter ermöglichen.

\section{Sternoklaviculargelenk}

Das Sternoklavikulargelenk ist funktionell ein Kugelgelenk mit einer oft unter-

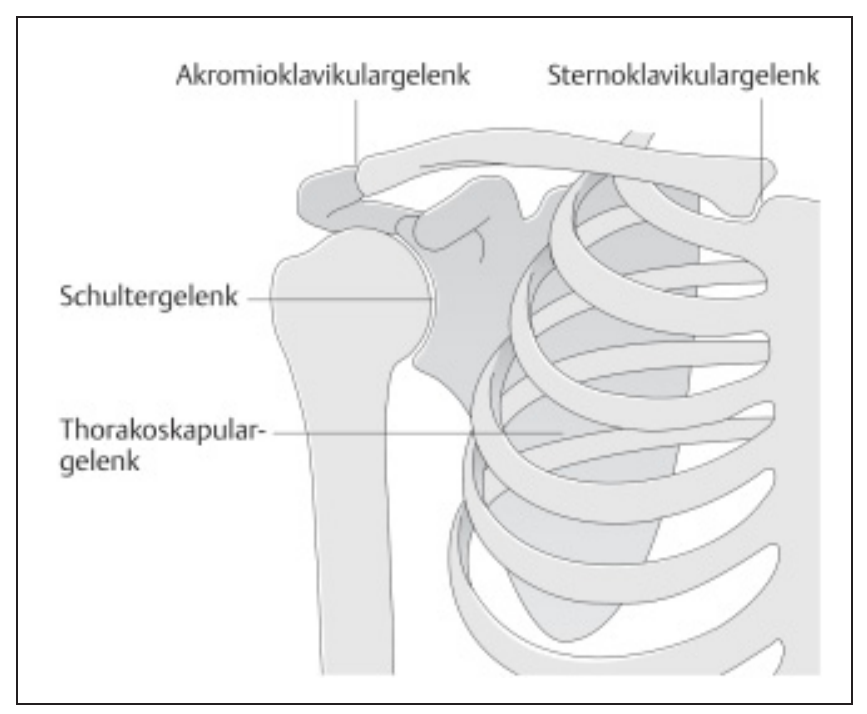

schätzten Beweglichkeit von 45 Grad in der Vertikalen, von 30 Grad in ventraldorsaler Richtung und einer Rotationsmöglichkeit von 45 Grad. Die belastungsfähige Verbindung zum Rumpf wird durch das an der ersten Rippe ansetzende sehr kräftige Lig. costoclaviculare hergestellt.

Degenerativ bedingte Beschwerden kommen an diesem Gelenk selten vor, obwohl im Alter häufig arthrotische Veränderungen nachweisbar sind. Verletzungen in Form einer ventralen oder dorsalen Luxation sind ebenfalls selten. Bei der dorsalen Luxation und der bei der operativen Versorgung von Verletzungen dieses Gelenks ist die Nähe zu den großen Gefäßen, Vena subclavia und Truncus brachiocephalicus zu beachten (Abb.2), welche mitverletzt sein können.

Bei Luxationen des Sternoklavikulargelenks nach dorsal, kann es zu Verletzungen der großen Gefäße im Thorax kommen.

Die Gefäßverletzung wird eventuell erst nach Reposition durch eine starke Blutung manifest, weil die Gefäße zuvor

Abb.1 Der große Bewegungsumfang der Schulter wird durch das Zusammenspiel von vier Gelenken erreicht: Sternoklavikulargelenk, Acromioclavikulargelenk, Schulterhauptgelenk und sog. „Thorakoskapulargelenk“. 


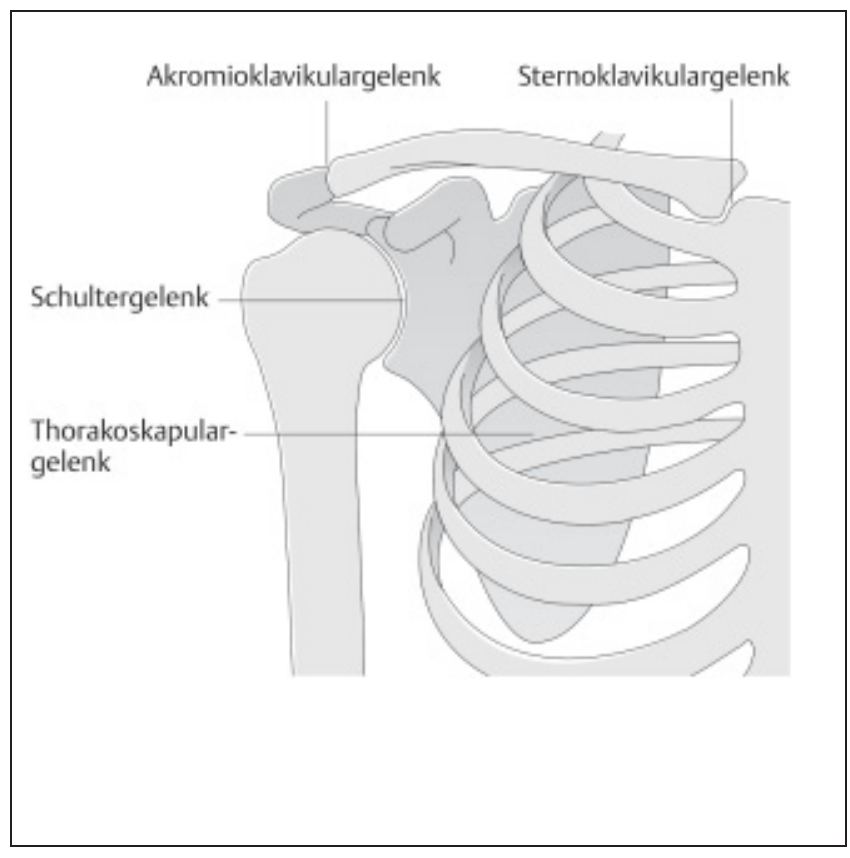

Abb. 2 Die großen Gefäße der oberen Thoraxappertur stehen in enger topographischer Beziehung zum Sternoklavikulargelenk.

Abb. 3 Das Schulterblatt ist über Ligamente, Muskeln und die Klavikula aufgehängt (modifiziert nach Neer).

mentären Strukturen tritt als dynamische Komponente die Aufhängung des Schulterblattes durch den Musculus trapezius und M. levator scapulae hinzu. In Zugrichtung nach kranial setzen an der Klavicula der Vorderrand des M. trapezius und der M. sternocleidomastoideus an und stellen ein Kräftegleichgewicht zu den vorgenannten Bandstrukturen her (Abb. 3).

\section{Akromioklavikulargelenk}

Das AC-Gelenk wird von den planen, faserknorpeligen Flächen des lateralen Klavikulaendes und des Akromions gebildet, zwischen die ein Discus articularis geschaltet ist. Die Beweglichkeit des AC-Gelenkes ist infolge der Fixierung des lateralen Klavikulaendes durch kräftige Bänder stark eingeschränkt.

Klinisch erfolgt die Hauptbewegung im AC-Gelenk als Rotation, was bei gelenküberbrückenden Osteosyntheseverfahren berücksichtigt werden muss.

Das größte Bewegungsausmaß erfährt die Rotation der Klavikula um ihre Längsachse, eine Tatsache, die bei der Auswahl von gelenksüberbrückenden Osteosyntheseverfahren an diesem Gelenk bedacht werden muss.

\section{Scapula}

Das Schulterblatt wird durch den Tonus der an ihm ansetzenden Muskeln gegen die Thoraxwand fixiert. Lähmungen führen zum klinisch bekannten Bild der Scapula alata. Drehbewegungen erfolgen durch das Zusammenspiel des M. serratus anterior und das M. trapezius, Vorund Rückführen der Scapula durch M. serratus und $\mathrm{M}$. rhomboideus.

Außer diesen an den Rändern des Schulterblatts ansetzenden Muskeln, entspringt von der durch die Spina scapulae unterteilten Rückfläche des Schulterblatts der M. infraspinatus der M. supraspinatus und der M. teres minor. Die Unterfläche des Schulterblatts nimmt mit breitem Ursprung der M. subscapularis ein.

durch das nach dorsal luxierte sternale Klavikulaende komprimiert sind.

\section{Klavikula}

Die Klavikula kann als ein durch Gelenke einstellbarer Aufhängemechanismus für die Skapula aufgefasst werden. Die Aufhängung erfolgt durch das Lig. coracocla- viculare, welches man in ein mediales Lig. conoideum und ein lateral gelegenes Lig. trapezoideum unterteilt. Letzteres Band kann bis $1 \mathrm{~cm}$ an das laterale Klavikulaende heranreichen, was bei AC-Gelenks-Resektionen beachtet werden sollte. Im Akromioklavikulargelenk wird die Verbindung zur Skapula durch das Lig. acromioclaviculare gesichert. $\mathrm{Zu}$ diesen liga-
Durch die Bewegungsmöglichkeit des Schulterblattes wird die Beweglichkeit der Schulter und damit die Positionierung der Hand im Raum wesentlich erweitert.

Das Schulterblatt besitzt zwei charakteristische nach ventral gerichtete Fort- 


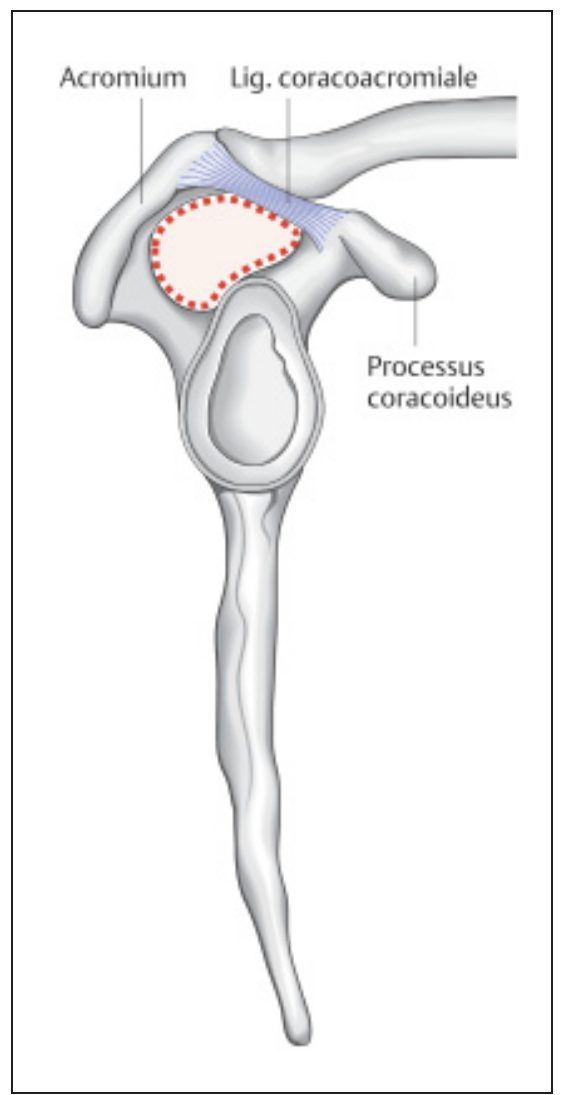

Abb. 4 Das Akromion, das korakoklavikuläre Band und der Prozessus coracoideus bilden den „Fornix humeri“.

sätze, Akromion und Processus coracoideus. Zwischen beiden Fortsätzen spannt sich das kräftige Lig. coracoacormiale aus. Zusammen mit den beiden Knochenvorsprüngen bildet es ein osteofibröses Dach für den Humeruskopf, die sogenannte Fornix humeri (Abb.4).

Das Lig. coracoacromiale wirkt als Puffer gegen eine Kranialverschiebung des $\mathrm{Hu}-$ meruskopfes und erlangt besondere Bedeutung, wenn die muskuläre Führung des Humeruskopfes beeinträchtigt ist, was besonders auch für die Situation nach Endoprothesenimplantation gilt.

Unter diesem osteofibrösen Dach verlaufen die flächigen Sehnen der Rotatorenmanschette wie in einem Kanal, der nach unten vom Humeruskopf eng begrenzt ist. Die Ausformung dieses Kanals ist ausschlaggebend für eine ungehinderte Gleitfunktion. Dabei kommt der Form des Akromions eine gewisse Bedeutung zu, da der Neigungswinkel des Akromions für die „Höhe des Kanals“ bestimmend ist (Abb.5). Sonderfälle, die ebenfalls zu einer Einengung des subakromialen Raums führen, sind die Nichtvereinigung der Akro-

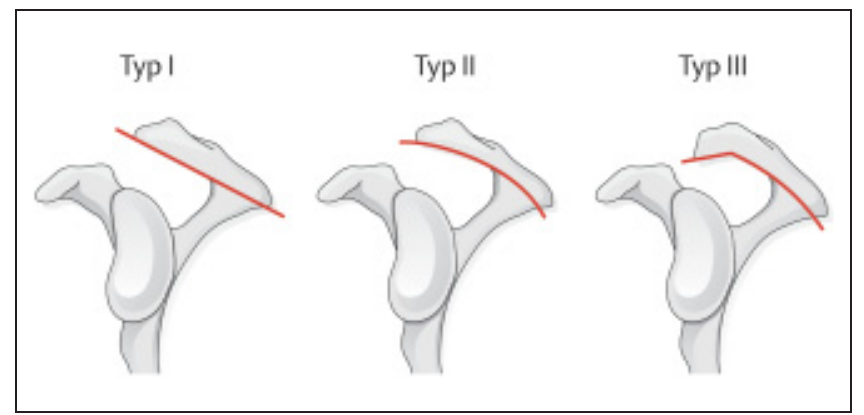

Abb. 5 Einteilung der Akromiontypen nach Bigliani.

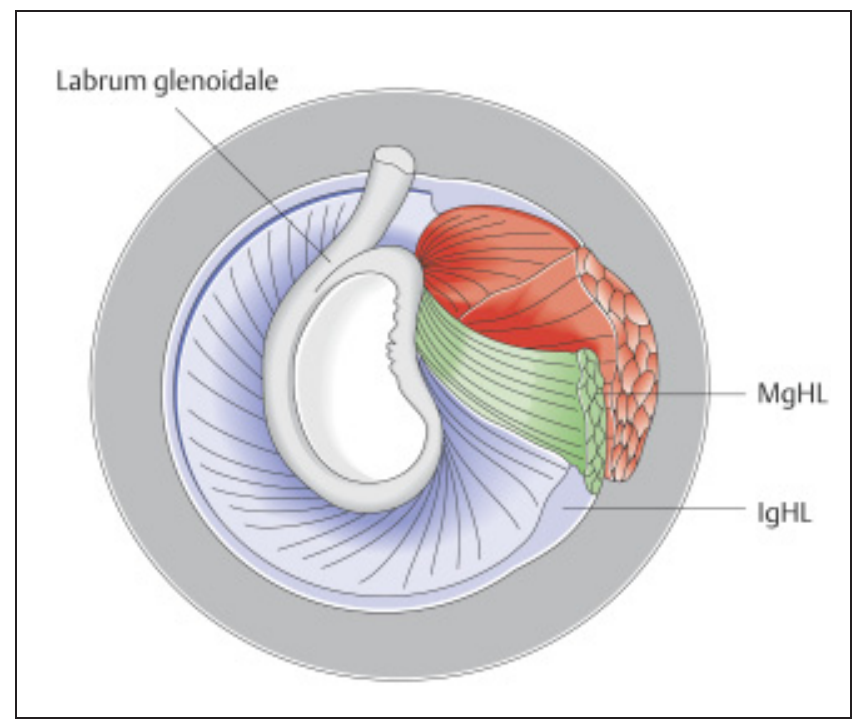

Abb. 6 Das inferiore glenohumerale Band setzt über das Labrum glenoidale am vorderen unteren Pfannenrand an.

mionepiphyse, mit wulstigen Auftreibungen der die Fuge begrenzenden knöchernen Akromioanteile, die Ausbildung eines Knochensporns am Ansatzpunkt des Lig. coracoacromiale und die Ausbildung von caudalen Exophyten bei der Arthrose des Akromioklavikulargelenks.

Formveränderungen des Akromions haben Bedeutung bei der Entstehung von Erkrankungen im subakromial Raum.

\section{Schultergelenk}

Betrachtet man das Schultergelenk, fällt das Missverhältnis zwischen der Größe des Humeruskopfs und der Gelenkpfanne des Schulterblattes auf. Das Verhältnis von Kopf zu Pfannendurchmesser wird auch als glenohumeraler Index bezeichnet. Der Humeruskopf ist gegenüber einer durch die Humeruskondylen gezogenen Geraden um 25 bis 40 Grad retroviert, die relativ flach ausgeprägte Pfanne weist ebenfalls individuell variable Neigungswinkel auf. Diese und weitere knöcherne Maße werden als Kriterien für die Gelenkstabilität verwendet, sind aber allein nicht ausschlaggebend, weil andere
Mechanismen entscheidend mit zur Stabilität beitragen müssen.

Ossäre Variationen am Oberarmkopf und an der Gelenkpfanne haben im Vergleich zu den ligamentären und muskulären Strukturen wenig Einfluss auf die Stabilität des Schultergelenks.

Die Gelenkkapsel ist entsprechend der großen Beweglichkeit nicht sehr straff und spannt sich erst mit zunehmender Abduktion des Arms an, wodurch dann die Rotationsbeweglichkeit in Abduktion wesentlich geringer ist als beim herunterhängenden Arm. In die Gelenkkapsel eingewoben sind Verstärkungen in Form der drei Ligamenta glenohumeralia (Ligamentum glenohumerale superius, medium, inferius, im engl. meist abgekürzt als SGHL, MGHL und IGHL). Die Bänder sind individuell sehr variabel ausgeprägt, teils kaum wahrnehmbar, teils strangartig ins Gelenk vorspringend. Funktionell am wichtigsten ist das inferiore Band, welches bei der ventralen Schulterluxation mit dem ventrokaudalen Labrum glenoidale vom Glenoid abgerissen wird (Bankartschaden, Abb.6). 
Das Ligementum glenohumerale inferior stellt das funktionell wichtigste Band in der Schultergelenkkapsel dar. Dieses Band wird mit dem Labrum glenoidale bei der vorderen Schulterluxation vom Glenoid abgerissen.

Als weitere Verstärkung der Gelenkkapsel ist das Lig. coracohumerale zu benennen, welches lateral am Proc. coracoideus entspringt und über die Bizepssehne hinwegziehend mit einem Schenkel am Tub. majus, hier zusammen mit der Supraspinatussehne und am Tub. minus ansetzt. Zusammen mit dem SGHL bildet das Lig. coracohumerale eine Art Ringband für die Bizepssehne. Aus diesen anatomischen Gegebenheiten wird klar, dass Schädigungen an der Supraspinatussehne auch Auswirkungen auf die Festigkeit dieses Ringbandsystems hat, dessen Beeinträchtigung zur Bizepssehnenluxation nach medial führen kann.

Die Gelenkpfanne erfährt durch das Labrum glenoidale eine bedeutende Vergrößerung und auch eine ausgeprägtere Konkavität, was klinische Bedeutung für die Stabilität des Schultergelenks hat.

Kranial strahlt die lange Bizepssehne in das Labrum ein, kaudal wird es durch Fasern der langen Trizepssehne verstärkt. Die Verbindung Labrum/lange Bizepssehne stellt einen Schwachpunkt dar, an dem es zu Labrumrissen kommen kann, welche als SLAP lesions bezeichnet werden.

Der Humeruskopf wird fast in seiner ganzen Ausdehnung kappenförmig von den Sehnen der M. teres minor, M. infraspinatus, M. supraspinatus und M. subscapularis als der Rotatorenmanschette umfasst (Abb. 7). Zwischen dem M. supraspinatus und M. subscapularis besteht eine muskuläre Lücke, die man als Rotatorenintervall bezeichnet, dem bei der nichttraumatischen Instabilität des Schultergelenks Bedeutung zukommt. Eine zweite Lücke besteht zwischen M. subscapularis und M. teres minor, der axilläre Rezessus. Die Muskeln der Rotatorenmanschette haben einerseits Aufgaben des Außenrotatoren (teres minor und infraspinatus), Abduktoren (M. supraspinatus) oder Innenrotator (M. subscapularis). Gemeinsame Hauptaufgabe dieser Muskeln ist jedoch den Humeruskopf in der Gelenkpfanne zu stabilisieren und so das Angreifen wesentlich stärkerer Muskeln wie etwa dem M. deltoideus als stärkstem Abduktor zu ermöglichen, ohne dass es zu einer Dislokation des Humeruskopfes kommt.

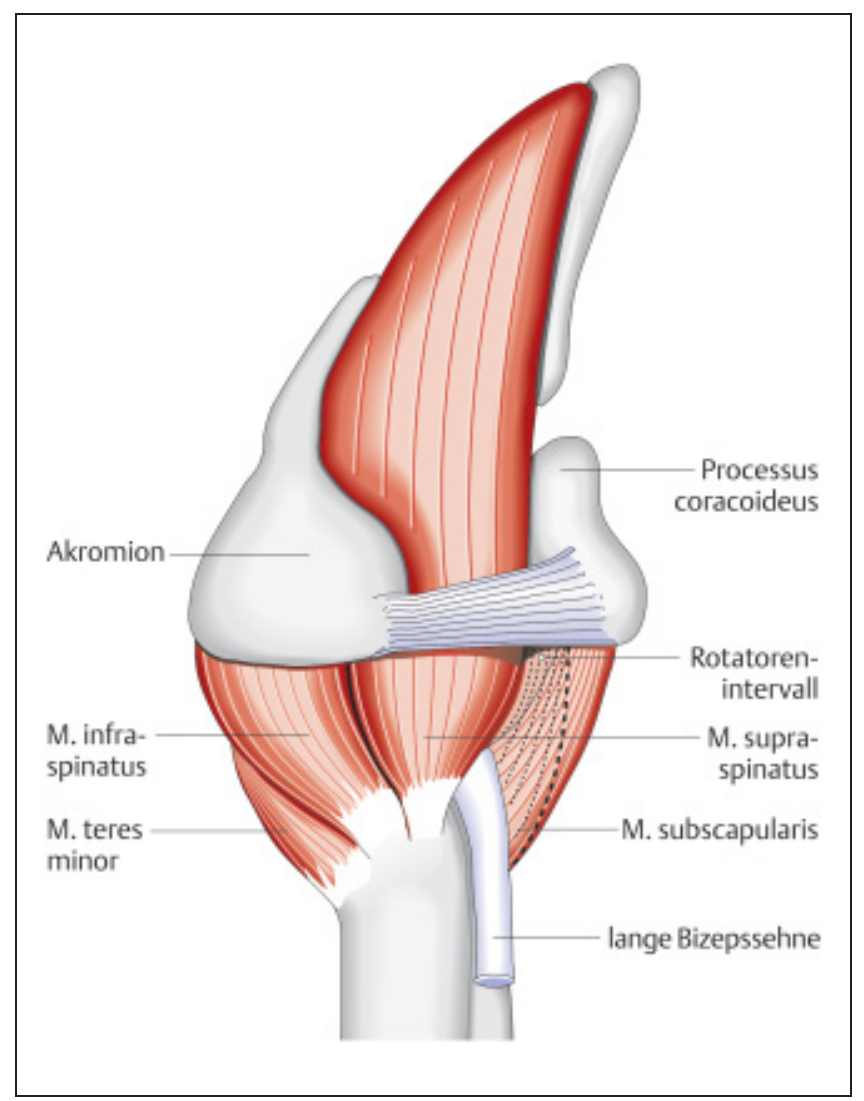

Abb. 7 Die Rotatorenmanschette legt sich kappenförmig über den Oberarmkopf.

Die Hauptaufgabe der Rotatorenmanschette besteht in der Stabilisierung des Humeruskopfes im Glenoid bei der kraftvollen Bewegung des Gelenks.

Eine solche Dislokation ist bei Rupturen der Rotatorenmanschette in Form eines Humeruskopfhochstands zu beobachten, wodurch der subakromiale Raum weiter eingeengt wird und die Schädigung der Manschette desto schneller voranschreitet. In diesem Zusammenhang muss erwähnt werden, dass auch der langen Bizepssehne durch ihren Verlauf vor und über dem Humeruskopf eine nach ventral und kranial stabilisierende Wirkung zukommt.

\section{Schleimbeutel}

Der große Bewegungsumfang des Schultergelenks bringt es mit sich, dass sich während des Bewegungsablaufs auch Gewebeschichten langstreckig gegeneinander gleiten müssen. Zu diesem Zweck sind um das Gelenk mehrere Schleimbeutel angeordnet. Der das Schultergelenk umhüllende $\mathrm{M}$. deltoideus ist gegen das Tuberculum majus und den Humeruskopf durch die Bursa subdeltoiden abgegrenzt. Häufig besteht von hier aus eine Verbindung zur Bursa subacromialis, welche die Sehnenplatte der Rotatorenmanschette gegen die Akromionunterfläche abpolstert (Abb. 8). Zwischen diesen beiden Bursae und dem Gelenk besteht normalerweise keine Verbindung. Die Bursa subacromialis, welche das Gleiten der Sehnen im subakromialen Raum begünstigen und erleichtern soll, kann im Fall einer Entzündung oder Verdickung selbst zur Verengung des Subakromial-

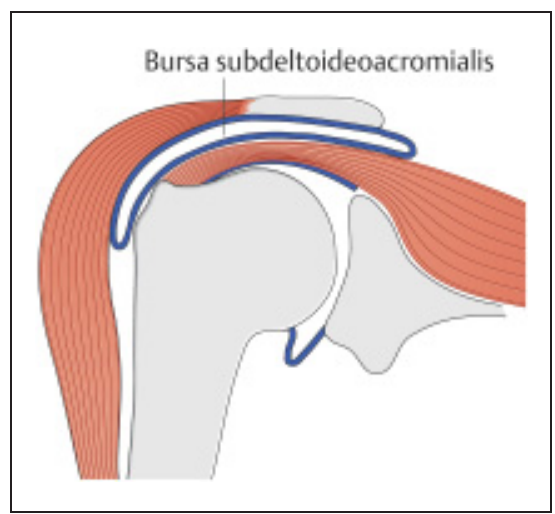

Abb. 8 Die Bursa subdeltoideoacromialis stellt die Verschiebeschicht zwischen Oberarmkopf und Supraspinatussehne einerseits und Muskulus deltoideus und Akromion andererseits dar. 
raums und damit $\mathrm{zu}$ einer Einklemmungssymptomatik (Impingement) führen. Die Schmerzsymptomatik bei Erkrankungen des subakromialen Raumes werden zumindest zum Teil durch die Entzündung dieser Bursa erklärt.

Entzündungen der Bursa subacromialis sind mitverantwortlich für die Schmerzsymptomatik bei Erkrankungen und Verletzungen im subakromialen Raum.

Weitere nicht mit dem Gelenk kommunizierende Bursen, welche selten klinische Bedeutung erlangen, finden sich am Proc coracoideus unter dem Ansatz des M. coracobrachialis und unterhalb der Sehne des M. infraspinatus. Allein die Bursa subtendinea Mi. subscapularis weist regelmäßig eine Verbindung zum Schultergelenk auf.

\section{Gefäße und Nerven}

Zur besseren Übersicht kann man die arterielle Gefäßversorgung der Schulter in einen dorsalen und einen ventralen Bereich unterteilen. Die dorsalen Partien des Schulterbereichs werden ausgehend von der A. subclavia über den Truncus thyreocervicalis von der A. suprascapularis versorgt. Es besteht eine Kollateralverbindung zur A. axillaris über die zur A. circumflexa scapulae und A. subscapularis.

Die aus der A. axillaris abgehende A. circumflexa humeri posterior tritt zusammen mit dem N. axillaris durch die laterale Achsellücke, tritt von hinten in Höhe des Collum chirurgicum an den Humerus und bildet hier mit der ebenfalls aus der A. axillaris entspringenden A. circumflexa humeri anterior einen Gefäßkranz zur Versorgung des Humeruskopfes. Wobei der ausgeprägteste Ast für die Versorgung des Oberarmkopfes die Arteria arcuata aus der Arteria circumflexa humeri anterior darstellt. Dieses Gefäß zieht im Sulcus der langen Bizepssehne nach kranial und versorgt den größten Teil des Oberarmkopfes. Für die Versorgung der Kopfkalotte bei Mehrsegmentbrüchen große Bedeutung haben Äste aus der Arteria circumflexa humeri posterior, die von dorsal in den Oberarmkopf einstrahlen (Abb.9).

Die Blutversorgung der Oberarmkopfkalotte erfolgt neben der von ventral kommenden Art. arcuata, auch über dorsal in den Kopf einstrahlende Gefäße.

Von ventral zieht die A. thorcoacromialis heran. Zur Schulter zieht aus diesem Ge-

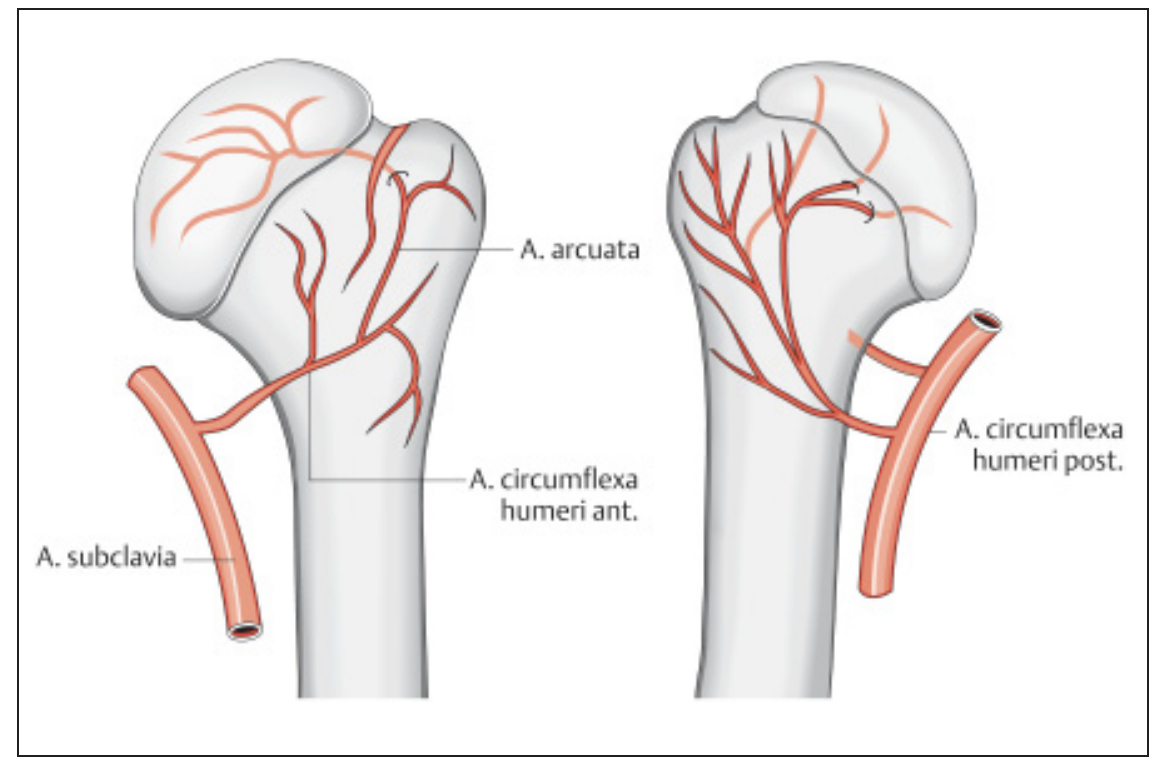

Abb.9 Die Durchblutung des Oberarmkopfes erfolgt durch Äste aus der Arteria circumflexa humeri anterior und posterior.

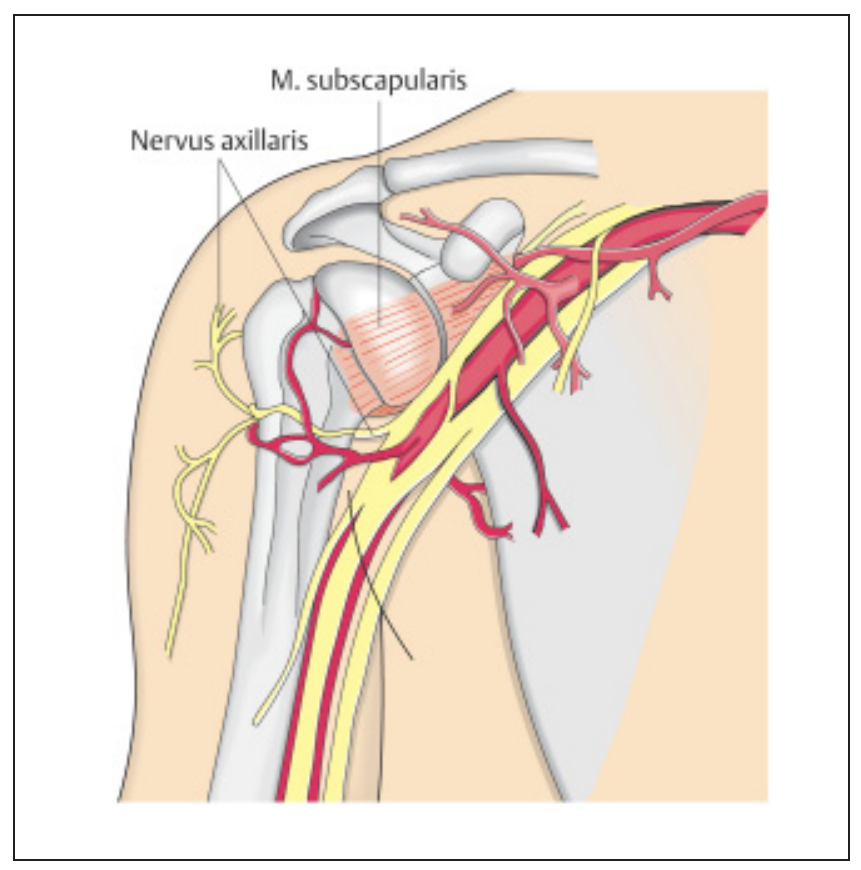

Abb.10 Der Nervus axillaris verläuft in enger Nachbarschaft zum Unterrand der Sehne des Muskulus subscapularis.

fäßstamm ein Ast zum M. deltoideus, und ein weiterer Ast, Ramus acromialis der von unten an das Akromion herantritt und an dieser Stelle beim Einkerben des Lig. coracoacromiale meist verletzt wird.

Die sensible Innervation der dorsalen und oberen Schulterabschnitte geschieht durch Äste des N. suprascapularis, an der Vorderseite durch Äste des N. musculocutaneus und N. subscapularis. Daran schließen sich nach kaudal/dorsal sensible Äste aus dem N. axillaris an.
In der Schulterchirurgie spielt der N. axillaris durch seine motorische Innervation von $\mathrm{M}$. deltoideus und $\mathrm{M}$. teres minor eine besondere Rolle. In seinem Verlauf ist er intraoperativ an zwei Stellen verletzungsgefährdet: Einmal am Unterrand der Sehne des M. subscapularis (Abb.10) bei der operativen Stabilisierung der Schulter und weiter peripher an der Lateralseite des Oberarms, wo er an der Unterfläche des Deltamuskels verläuft. Beim antero-lateralen Zugang zum Subakromialraum darf deshalb die Länge des gesplitteten $\mathrm{M}$. deltoideus $5 \mathrm{~cm}$ nicht 


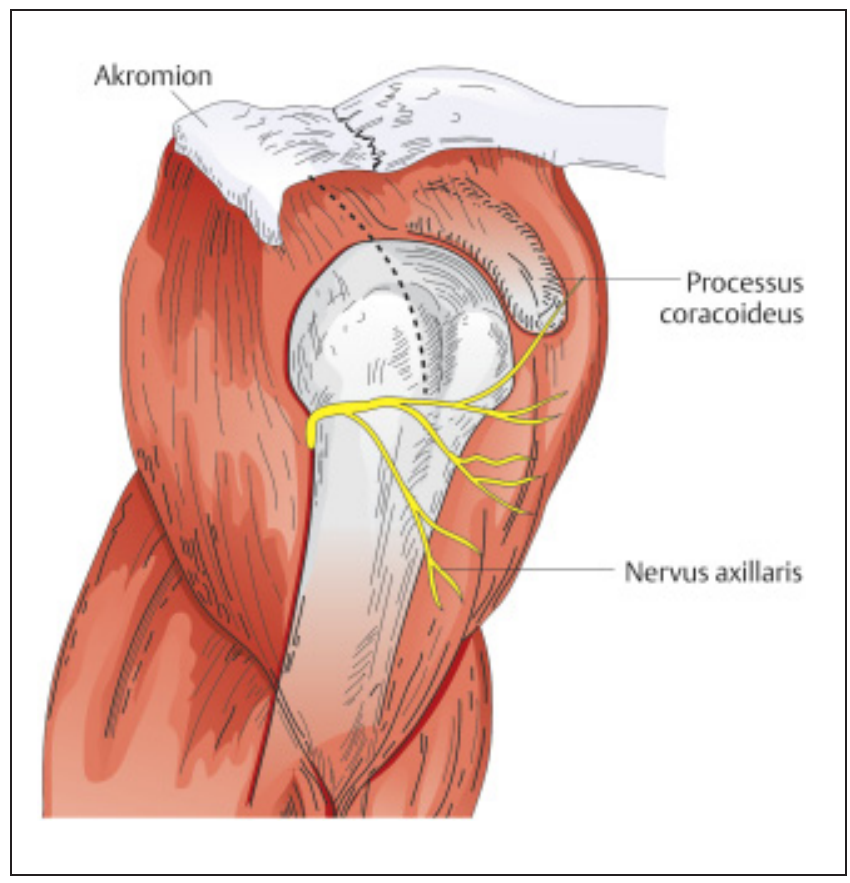

Abb.11 Am lateralen Oberarm verläuft der Nervus axillaris an der Unterfläche des Muskulus deltoideus im Abstand vom Akromion von etwa $5 \mathrm{~cm}$.

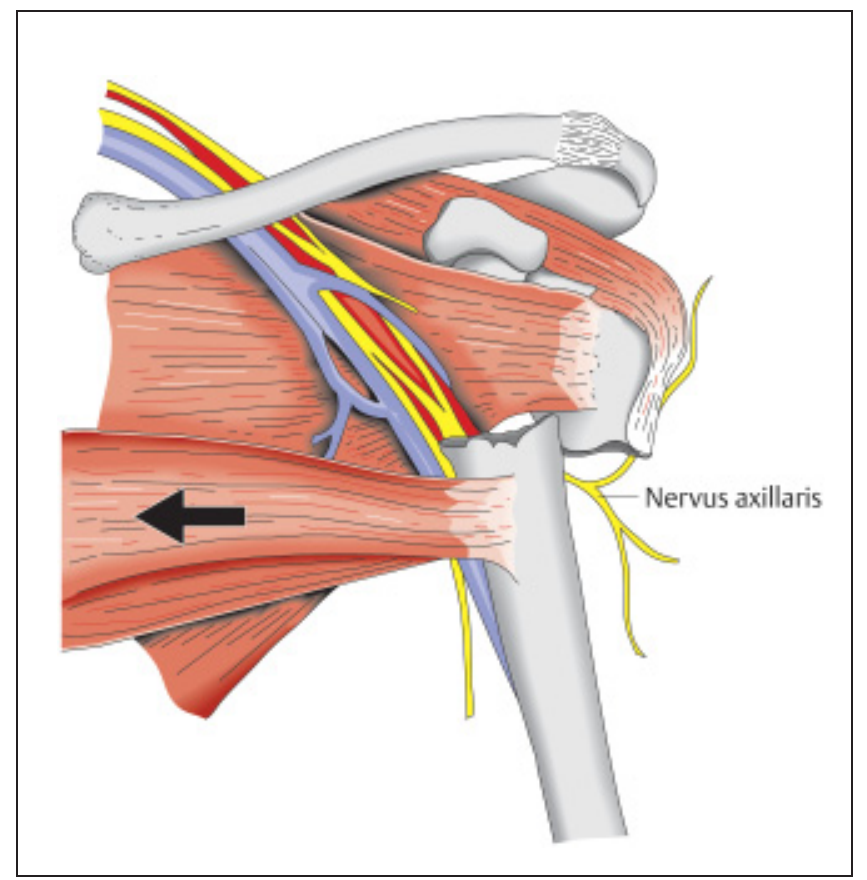

Abb.12 Aufgrund der topographischen Beziehungen kommt bei den Frakturen im chirurgischen Hals neben dem Nervus axillaris auch das medial gelegene Gefäßnervenbündel zum Arm in Gefahr. überschreiten (Abb.11). Des Weiteren kommt der Nerv bei Frakturen im Collum chirurgicum in Gefahr, da er beim Durchtritt durch die laterale Achsellücke in dieser Höhe relativ nahe am Oberarmknochen verläuft (Abb.12).
In diesem Zusammenhang sei auch darauf hingewiesen, dass sowohl der Verlauf des N. axillaris, als auch des Plexus brachialis und der Axillargefäße von der Gelenkstellung abhängig ist, welche infolge ihrer Verschieblichkeit bei Abduktion des Armes wesentlich näher an das Schultergelenk heranrücken.

\section{Literatur}

1 Rockwood and Matson. The Shoulder. $2^{\text {nd }}$ Ed. Vo. 1 Saunders Philadelphia 1998

2 Neer CS, Demarest RJ. Shoulder reconstruction. Saunders Philadelphia 1990

3 Habermeyer P. Schulterchirurgie. Urban und Fischer, München 2002

${ }^{4}$ Waldeyer A. Anatomie des Menschen Bd. 2. Walter De Gruyter, Berlin 1975

\section{Dr. med. Ulrich Rumschilk Oberarzt \\ Dr. med. Bernd Wittner Chefarzt}

Unfallchirurgische Abteilung Hohenloher Krankenhaus Stettener Straße 32

D-74653 Künzelsau 